\title{
Determination of the strange quark mass from Cabibbo-suppressed tau decays with resummed perturbation theory in an effective scheme
}

\author{
J.G. Körner ${ }^{1}$, F. Krajewski ${ }^{1}$, A.A. Pivovarov ${ }^{1,2}$ \\ ${ }^{1}$ Institut für Physik, Johannes-Gutenberg-Universität, \\ Staudinger Weg 7, D-55099 Mainz, Germany \\ ${ }^{2}$ Institute for Nuclear Research of the \\ Russian Academy of Sciences, Moscow 117312
}

\begin{abstract}
We present an analysis of the $m_{s}^{2}$-corrections to Cabibbo-suppressed $\tau$ lepton decays employing contour improved resummation within an effective scheme which is an essential new feature as compared to previous analyses. The whole perturbative QCD dynamics of the $\tau$-system is described by the $\beta$-function of the effective coupling constant and by two $\gamma$-functions for the effective mass parameters of the strange quark in different spin channels. We analyze the stability of our results with regard to high-order terms in the perturbative expansion of the renormalization group functions. A numerical value for the strange quark mass in the $\overline{\mathrm{MS}}$ scheme is extracted $m_{s}\left(M_{\tau}\right)=130 \pm 27_{\exp } \pm 9_{\text {th }} \mathrm{MeV}$. After running to the scale $1 \mathrm{GeV}$ this translates into $m_{s}(1 \mathrm{GeV})=176 \pm 37_{\exp } \pm 13_{\text {th }} \mathrm{MeV}$.
\end{abstract}

PACS: 11.10.Hi, 12.38.-t, 13.35.DX, 14.65.Bt

Keywords: resummation, tau decays, strange quark mass 


\section{Introduction}

The $\tau$-system offers the possibility to confront QCD with experiment in the low energy region. The high precision of experimental data and good accuracy of theoretical results make $\tau$-physics an important testing ground for QCD [1, 2, 3]. Theoretically the observables of the $\tau$-system are related to the moments of the spectral density of a correlator of hadronic currents which can be reliably calculated within perturbation theory [4, 5, 6]. Therefore, the $\tau$-system observables were extensively studied during the past few years within the operator product expansion (OPE) which is a general approach to analyzing the properties of current correlators [7, 8, 9, 10, 11]. The perturbation theory (PT) series in QCD appear to be asymptotic and the ultimate accuracy they can provide depends on the concrete numerical value of the expansion parameter - the strong coupling constant $\alpha_{s}(E)$ at a relevant energy $E$. This limits the theoretical accuracy which can be obtained within the finite order perturbation theory (FOPT) analysis. In the case of the $\tau$-system the strong coupling constant $\alpha_{s}\left(M_{\tau}\right)$ is not small at the scale of the $\tau$ lepton mass $M_{\tau}$ which can lead to an asymptotic growth of terms of the perturbation theory series already at a rather low (third-fourth) order of PT expansion. Judging from the analysis of the moments of the hadronic spectral density in the finite energy interval $\left(0, M_{\tau}^{2}\right)$ within finite order perturbation theory there are strong indications that the ultimate theoretical accuracy for the $\tau$ lepton decay observables has already been reached at next-to-nextto-next-to-leading order $\left(\mathrm{N}^{3} \mathrm{LO}\right)$ or $\alpha_{s}^{3}$ which is the highest order of PT expansions presently available [12]. The convergence behavior of the perturbation series for the $\tau$ lepton observables depends on the region of the spectral density which is being

probed: if the low energy region is suppressed (as for high moments of the spectral density) the asymptotic limit of the series moves to higher order terms. The expansion of the correlator in $m_{s}^{2}$ makes the explicit convergence of the PT series for the 
coefficient functions of consecutive $m_{s}^{2}$ corrections slower [13]. The reason for such a behavior is quite obvious - higher order terms of $m_{s}^{2}$ expansion of the correlator are more sensitive to the low energy region of integration in Feynman diagrams that is not described by perturbation theory. Thus, the PT expansions for observables in the $\tau$-system seem to be at the edge of asymptotic growth in the $\mathrm{N}^{3} \mathrm{LO}$. At the same time, the present accuracy of experimental data for some observables of the $\tau$-system is already comparable with the ultimate theoretical accuracy reachable in FOPT [2, 3]. This raises the problem of obtaining more precise theoretical formulas. Higher order terms of FOPT (though they are very desirable and provide additional information) will not give more precise results for PT series if the asymptotic limit is already reached and must simply be discarded in FOPT applications. To catch up with improving experimental accuracy it is necessary to find a way of interpretation of the perturbation theory series that allows one to reach a theoretical precision comparable to that of the experimental data. One possibility to extract numerical results from a perturbation theory series which is explicitly divergent at finite orders is to apply a resummation procedure (see e.g. [14). This is more sophisticated than just summing the consecutive terms of the perturbative expansion up to some finite order but it requires some knowledge (or assumptions) about the behavior of infinite number of terms of the expansion. The choice of a resummation procedure is not unique and there are many ways to resum or improve the convergence of an asymptotic series e.g. [15, 16]. We think that there are two important criteria for the choice of an appropriate resummation procedure for perturbation theory series of physical observables: the renormalization group structure of the PT series should be respected and the definition of the effective parameters used for the description of the observables should be physically motivated. Led by these two criteria we use contour improved perturbation theory (CIPT) [10, [1] (see also [18]) in an effective scheme 
(not $\overline{\mathrm{MS}}$ ) to resum perturbation theory contributions in all orders. Within CIPT, the procedure of resummation is, however, not unique. A freedom in the choice of different renormalization schemes still remains within CIPT, which will affect the final numerical results [19]. We choose an effective scheme which we consider to be natural and the simplest one for the $\tau$-system. Note that the choice of an adequate scheme is also dictated by the way the system is to be described. In the FOPT analysis of the moments, the contour integration is completely (mathematically) equivalent to the integration along the physical cut. Therefore, the basic object for FOPT analysis is the PT spectral density of the correlator which determines the effective coupling in this procedure [12]. In CIPT, the correlator is the basic perturbation theory object which is naturally normalized in Euclidean domain. Within the effective scheme approach all PT corrections to the correlator are absorbed into the definition of the effective parameters of the system [20, 21, 22, 23, 24, 25]. In the massless case the effective coupling constant is the only relevant parameter. If the $m_{s}$-corrections to the correlator are included then two additional parameters $m_{q}^{2}$ and $m_{g}^{2}$ related to mass corrections to different spin structures of the correlator should be considered. These parameters are perturbatively related to a finite strange quark mass. In the massless case the effective scheme resummation analysis along these lines was done in [19]. In this paper the resummation analysis in the effective scheme is extended to $m_{s}^{2}$ corrections. An analysis of the $m_{s}^{2}$ corrections within CIPT in the $\overline{\mathrm{MS}}$ scheme was previously performed in refs. [26, 27]. Our results of resummation within the effective scheme approach confirm the main conclusions of the paper 26] and are consistent with the results of ref. [27] though the direct comparison is a bit complicated because we use a different parameterization of the relevant observables. We also use different moments for the final determination of a numerical value for $m_{s}$ as compared to ref. [27 - the detailed discussion of our choice is given in the text. The uncertainty 
of our new results is smaller than the conservative error bars given in ref. [26] on the base of an analysis of the explicit convergence of PT series. In order to get an understanding of the reliability of our present procedure we evaluate the stability of our results with regards to higher order corrections to the renormalization group functions.

The paper is organized as follows. In Sect. 2 we set up the stage for resummation in an effective scheme. We define our basic quantities and calculate their respective anomalous dimensions (beta-functions) that determine the running along the integration contour. The known results of PT calculation in the $\overline{\mathrm{MS}}$ scheme are used but the expressions for the corresponding renormalization group ( $R G$ ) functions of effective parameters are new and specific for the effective scheme defined in the paper. In Sect. 3 we present the results of theoretical calculations of the moments of the spectral density of $\tau$ decays in the effective scheme. The PT expressions are given in the effective scheme which is a new result. We introduce the effective mass parameters for the channels with spin 1 and spin 0 and give explicit formulas expressing these parameters through the standard $\overline{\mathrm{MS}}$ mass $m_{s}$ in a resummed form. Some nonPT corrections are taken at the leading order only and are therefore scheme independent: they coincide with the results already published in the literature (see, e.g. 27]). In Sect. 4 we extract a numerical value for the strange quark mass from experimental data and compare our results with conclusions of earlier analyses. Sect. 5 gives our conclusion. In an Appendix the RG functions of the effective scheme are explicitly given through the coefficient of the $\overline{\mathrm{MS}}$-scheme RG functions known from the literature. 


\section{Resummation in an effective scheme}

In the first step of our analysis of the $\tau$-system we define an effective scheme in which all higher order PT corrections to the correlator of hadronic currents are absorbed into the effective coupling and two effective mass scales, or two coefficient functions of mass corrections. If such a scheme is used for describing the $\tau$-system the only perturbative objects are one effective $\beta$-function for the coupling constant and two effective $\gamma$ functions for the coefficient functions of mass corrections. Using these three functions we determine the evolution of the effective coupling and of the two coefficient functions of mass corrections on the contour in the complex plane of squared momentum. Within our procedure the $\beta$ - and $\gamma$-functions are treated as exact functions which is a standard understanding of renormalization group ( $R G)$ summation. Having the explicit solutions for the running coupling and the mass coefficient functions in hand we determine observables such as the moments of the decay rate simply by integrating the coefficient functions with a weight function specific to a chosen observable.

\subsection{Definition of an effective scheme}

The basic theoretical quantity for describing the $\tau$ semileptonic decays is the correlator of two hadronic currents

$$
\Pi_{\mu \nu}(q)=i \int d x e^{i q x}\left\langle T j_{\mu}(x) j_{\nu}^{\dagger}(0)\right\rangle=\frac{N_{c}}{6 \pi^{2}}\left(q_{\mu} q_{\nu} \Pi_{q}\left(q^{2}\right)+g_{\mu \nu} \Pi_{g}\left(q^{2}\right)\right)
$$

with $j_{\mu}(x)=\bar{u} \gamma_{\mu}\left(1-\gamma_{5}\right) s$. Here $\Pi_{q}\left(q^{2}\right)$ and $\Pi_{g}\left(q^{2}\right)$ are scalar invariant functions, $N_{c}=3$ is the number of colors in QCD. We work within QCD with three light quarks. The correlator is normalized to unity in the leading (parton model) approximation with massless quarks. By expanding $\Pi_{q}\left(q^{2}\right)$ and $\Pi_{g}\left(q^{2}\right)$ in terms of powers of $m_{s}^{2} / q^{2}$ and keeping only the first order term in this expansion one has

$$
\Pi_{q}\left(q^{2}\right)=\Pi\left(q^{2}\right)+3 \frac{m_{s}^{2}}{q^{2}} \Pi_{m q}\left(q^{2}\right),
$$




$$
\Pi_{g}\left(q^{2}\right)=-q^{2} \Pi\left(q^{2}\right)+\frac{3}{2} m_{s}^{2} \Pi_{m g}\left(q^{2}\right)
$$

where $\Pi\left(q^{2}\right)$ is an invariant function already known from the mass zero case. The functions $\Pi_{i}\left(Q^{2}\right)\left(i=m_{q}, m_{g}\right)$ with $Q^{2}=-q^{2}$ are computable in perturbation theory in the deep Euclidean region $Q^{2} \rightarrow \infty$. The results of perturbation theory calculations for the correlator given in eqs. (2, 3) were obtained in refs. [28, 29, 30, 31] and have already been used in the FOPT analysis [12].

We define new effective quantities $a, m_{q}^{2}, m_{g}^{2}$ such that all information from perturbation theory calculations for the functions $\Pi\left(Q^{2}\right), \Pi_{m q, m g}\left(Q^{2}\right)$ is absorbed into the evolution of these new quantities which is determined by the effective $\beta$ - and $\gamma$-functions. For the mass corrections we introduce two different coefficient functions (we sometimes call them the effective mass parameters) because the correlator in eq. (1) is not transverse if corrections of the order $m_{s}^{2}$ are taken into account. Our definitions of effective quantities $a, m_{q}^{2}, m_{g}^{2}$ are

$$
\begin{aligned}
-Q^{2} \frac{d}{d Q^{2}} \Pi\left(Q^{2}\right) & =1+a\left(Q^{2}\right) \\
-m_{s}^{2}\left(M_{\tau}^{2}\right) Q^{2} \frac{d}{d Q^{2}} \Pi_{m g}\left(Q^{2}\right) & =m_{g}^{2}\left(M_{\tau}^{2}\right) C_{g}\left(Q^{2}\right) \\
m_{s}^{2}\left(M_{\tau}^{2}\right) \Pi_{m q}\left(Q^{2}\right) & =m_{q}^{2}\left(M_{\tau}^{2}\right) C_{q}\left(Q^{2}\right) .
\end{aligned}
$$

Here $C_{q, g}\left(Q^{2}\right)$ are coefficient functions of mass corrections. They are conveniently normalized by the requirement $C_{q, g}\left(M_{\tau}^{2}\right)=1$.

In terms of the $\overline{\mathrm{MS}}$ scheme quantities $\alpha_{s} \equiv \alpha_{s}\left(M_{\tau}^{2}\right)$ and $m_{s} \equiv m_{s}\left(M_{\tau}^{2}\right)$ the effective parameters in eq. (4) read

$$
\begin{aligned}
a\left(M_{\tau}^{2}\right) & =\frac{\alpha_{s}}{\pi}+k_{1}\left(\frac{\alpha_{s}}{\pi}\right)^{2}+k_{2}\left(\frac{\alpha_{s}}{\pi}\right)^{3}+k_{3}\left(\frac{\alpha_{s}}{\pi}\right)^{4}+\mathcal{O}\left(\alpha_{s}^{5}\right) \\
m_{g}^{2}\left(M_{\tau}^{2}\right) & =m_{s}^{2}\left(M_{\tau}^{2}\right)\left(1+\frac{5}{3} \frac{\alpha_{s}}{\pi}+k_{g 1}\left(\frac{\alpha_{s}}{\pi}\right)^{2}+k_{g 2}\left(\frac{\alpha_{s}}{\pi}\right)^{3}+\mathcal{O}\left(\alpha_{s}^{4}\right)\right) \\
m_{q}^{2}\left(M_{\tau}^{2}\right) & =m_{s}^{2}\left(M_{\tau}^{2}\right)\left(1+\frac{7}{3} \frac{\alpha_{s}}{\pi}+k_{q 1}\left(\frac{\alpha_{s}}{\pi}\right)^{2}+k_{q 2}\left(\frac{\alpha_{s}}{\pi}\right)^{3}+\mathcal{O}\left(\alpha_{s}^{4}\right)\right) .
\end{aligned}
$$

Numerical values for the coefficients $k_{3}, k_{q 2}$ are unknown though their estimates within 
various approaches can be found in the literature. We have written explicitly first coefficients for mass corrections, $k_{g 0}=5 / 3, k_{q 0}=7 / 3$. Further references concerning numerical values for the known coefficients $k_{1,2}, k_{g 1, g 2}, k_{q 1}$ are given in Appendix.

\subsection{Running of the effective coupling $a\left(Q^{2}\right)$ and the mass coefficient functions $C_{q, g}\left(Q^{2}\right)$}

The behavior of the effective coupling $a\left(Q^{2}\right)$ and the coefficient functions of the mass parameters $C_{q, g}\left(Q^{2}\right)$ is determined by the effective beta and gamma functions. The defining RG equations for the evolution of these effective quantities are

$$
\begin{aligned}
Q^{2} \frac{d}{d Q^{2}} a\left(Q^{2}\right) & =\beta(a), \\
Q^{2} \frac{d}{d Q^{2}} C_{g}\left(Q^{2}\right) & =2 \gamma_{g}(a) C_{g}\left(Q^{2}\right), \\
Q^{2} \frac{d}{d Q^{2}} C_{q}\left(Q^{2}\right) & =2 \gamma_{q}(a) C_{q}\left(Q^{2}\right) .
\end{aligned}
$$

The RG functions $\beta(a)$ and $\gamma_{g, q}(a)$ describing the evolution of the parameters in the effective scheme can be expressed through the $\overline{\mathrm{MS}}$ scheme RG functions (the standard $\beta$-function of the coupling constant and the mass anomalous dimension $\gamma$ ) using the relations in eqs. (4, 5:6,7). The explicit formulas of such a RG transformation are given in Appendix. Up to the relative order $\alpha_{s}^{3}$ the RG functions $\beta\left(\alpha_{s}\right)$ and $\gamma\left(\alpha_{s}\right)$ in the $\overline{\mathrm{MS}}$ scheme have been calculated in refs. [32, 33, 34]. Under the RG transformation eqs. (5,607) the $\overline{\mathrm{MS}}$ scheme $\beta$ - and $\gamma$-functions are transformed into the effective $\beta$ - and $\gamma$-functions (8,9,10). Note that first two coefficients of the $\beta$-function $\beta_{0}, \beta_{1}$ and first coefficient of the $\gamma$-function $\gamma_{0}$ are invariant under RG transformations. Numerically the RG functions of the effective parameters describing the $\tau$-system up to the order $m_{s}^{2}$ are given by

$$
\beta(a)=-a^{2}\left(2.25+4 a+11.79 a^{2}+a^{3}\left(-76.36+4.5 k_{3}\right)\right)
$$




$$
\begin{aligned}
& \gamma_{g}(a)=-a\left(1+4.027 a+17.45 a^{2}+a^{3}\left(249.59-k_{3}\right)\right) \\
& \gamma_{q}(a)=-a\left(1+4.78 a+32.99 a^{2}+a^{3}\left(-252.47-k_{3}+3.38 k_{q 2}\right)\right) .
\end{aligned}
$$

For the effective coupling in eq. (四) we use the numerical value $a\left(M_{\tau}^{2}\right)=0.1445$ [35] extracted from the $\tau$ decay rate into nonstrange particles within the effective scheme resummation procedure described in ref. [19]. This value corresponds to the $\overline{\mathrm{MS}}$ scheme value of the coupling constant $\alpha_{s}\left(M_{\tau}^{2}\right)=0.343$ that is a bit larger than the most recent result obtained in FOPT analysis of the $\tau$ decay rate into nonstrange particles [35]. The PT series for the effective $\beta$-function in eq. (11) explicitly converges well at the numerical value $a\left(M_{\tau}^{2}\right)=0.1445$. If the coefficient $k_{3}$ lies in the range $0<k_{3}<50$ (which is a conservative estimate based on a number of predictions) the $a^{3}$ coefficient in eq. (11) will not be extremely large but nevertheless the $\beta$-function (11) shows asymptotic growth in the $\mathrm{N}^{3} \mathrm{LO}$ for $k_{3}>35$. The $\gamma_{g}$-function in eq. (12) behaves worse than the $\beta$-function but still the explicit convergence persists up to the NNLO at $a\left(M_{\tau}^{2}\right)=0.1445$. The $\mathrm{N}^{3} \mathrm{LO}$ correction will show asymptotic growth for values of $k_{3}$ smaller than 129 . The $\gamma_{q}$-function in eq. (13) has already shown an asymptotic growth in the NNLO which will limit the precision of our results. The $\beta$ - and $\gamma_{g}$-function will show asymptotic growth in the $\mathrm{N}^{3} \mathrm{LO}$ because no choice of $k_{3}$ can make them convergent simultaneously. This confirms the conclusions of ref. [12] where the asymptotic growth for the FOPT expressions of the moments of the spectral density has been found in $\mathrm{N}^{3} \mathrm{LO}$ independently of the choice of the numerical value for $k_{3}$. In other words, the evolution of the effective parameters for the $\tau$-system is too different to be handled by the FOPT expressions for the RG functions in the $\mathrm{N}^{3} \mathrm{LO}$.

The running of the effective coupling and the mass coefficient functions $C_{q, g}\left(Q^{2}\right)$ along the contour in the complex plane of momentum squared is determined by the renormalization group equations. It is convenient to choose a circular contour in the 
complex $Q^{2}$-plane and to parameterize it by the relation $Q^{2}=M_{\tau}^{2} e^{i \phi},-\pi<\phi<\pi$ which leads to the differential equations for the coupling constant

$$
-i \frac{d}{d \phi} a(\phi)=\beta(a(\phi)), \quad a(\phi=0)=a\left(M_{\tau}^{2}\right)
$$

and for the coefficient functions $C_{n}(\phi)$

$$
-i \frac{d}{d \phi} C_{n}(\phi)=2 \gamma_{n}(a(\phi)) C_{n}(\phi), \quad C_{n}(\phi=0)=1
$$

with $n=q, g$. The solution to the differential equation for the running mass in terms of the coefficient functions $C_{n}(\phi)$ in eq. (15) can be expressed through the integral

$$
C_{n}(\phi)=\exp \left(2 i \int_{0}^{\phi} \gamma_{n}(a(\chi)) d \chi\right) \text {. }
$$

The initial values for $a(\phi)$ and $C_{n}(\phi)$ are fixed at $Q^{2}=M_{\tau}^{2}$ or $\phi=0$. All corrections stemming from higher order perturbative terms are absorbed into the $\beta$ - and $\gamma$ function coefficients if the effective scheme is used as it is defined in eq. (团). The solutions to the differential equations for the coupling $a(\phi)$ (eq. (14)) and for the coefficient function $C_{q}(\phi)$ (eq. (15)) are shown in Figs. (11,2). The effective coupling $a(\phi)$ does not change much uniformly on the contour when higher order corrections of the $\beta$-function are included. Especially the change from NLO to NNLO is rather small. The behavior of the coefficient function $C_{g}(\phi)$ is rather similar to that of the effective coupling $a(\phi)$ as one can expect from the structure of PT series for $\gamma_{g}(a)$ in eq. (12) in comparison with the $\beta$-function in eq. (11). The function $C_{g}(\phi)$ appears to converge uniformly in the interval $|\phi|<\pi$ when going from NLO to NNLO. The function $C_{q}(\phi)$ (Fig. (2)) does not converge uniformly on the contour because of the slow convergence of the $\gamma_{q}$-function (eq. (13)) in consecutive orders of PT expansion. 

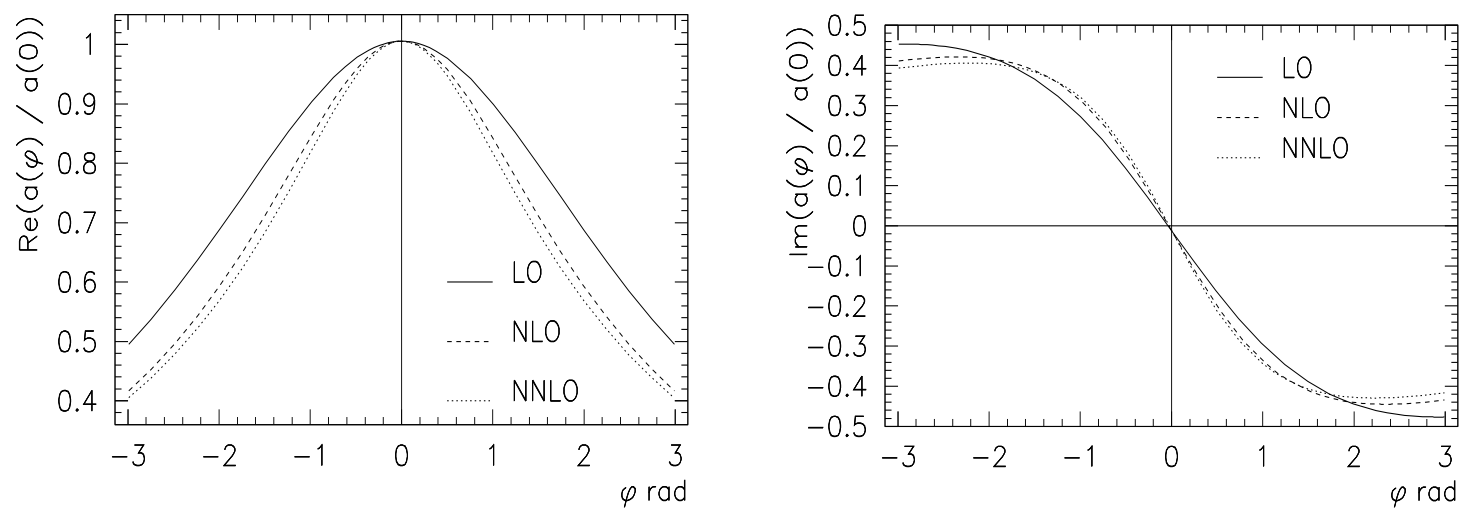

Figure 1: Running of the effective coupling $a(\phi)$ on a circular contour in the complex plane calculated at LO, NLO and NNLO (left: real part; right: imaginary part)
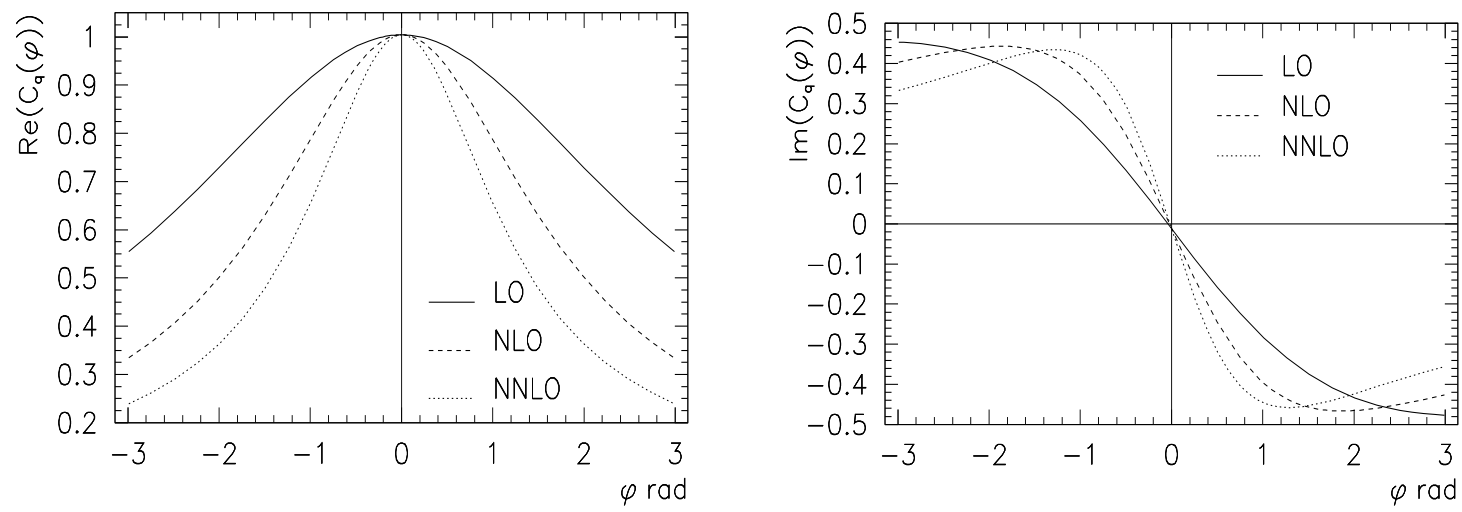

Figure 2: Running of the mass coefficient function $C_{q}(\phi)$ on a circular contour in the complex plane calculated at LO, NLO and NNLO (left: real part; right: imaginary part)

\subsection{Resummation}

We use the direct generalization of the resummation procedure described for the massless case in ref. [19]. We treat the renormalization group functions $\beta(a)$ and $\gamma_{n}(a)$ in any fixed order of PT (LO, NLO and NNLO) as exact functions and solve exactly (though numerically) the differential equations $(14,15)$ for the running parameters $a(\phi)$ and $C_{q, g}(\phi)$ on a circular contour in the complex plane. 
Having the solutions for the running parameters we calculate numerical values for the theoretical expressions of the observables in the $\tau$-system. The observables of interest are expressed through the moments of the spectral density of the basic correlator given in eq. (11). For the massless part of the correlator $\Pi\left(q^{2}\right)$ given in eqs. (2, 3) we define moments of the spectral density by the relation

$$
\begin{aligned}
M(n) & =(n+1) \frac{i}{2 \pi} \oint \Pi\left(q^{2}\right)\left(\frac{q^{2}}{M_{\tau}^{2}}\right)^{n} \frac{d q^{2}}{M_{\tau}^{2}} \\
& =1+(-1)^{n} \frac{1}{2 \pi} \int_{-\pi}^{\pi} e^{i(n+1) \phi} a(\phi) d \phi+\frac{1}{2 \pi} \int_{-\pi}^{\pi} a(\phi) d \phi .
\end{aligned}
$$

For the mass correction related to the $g$-part of the correlator the expressions for the moments are very similar. One has

$$
M_{g}(n)=\frac{(-1)^{n}}{2 \pi} \int_{-\pi}^{\pi} e^{i(n+1) \phi} C_{g}(\phi) d \phi+\frac{1}{2 \pi} \int_{-\pi}^{+\pi} C_{g}(\phi) d \phi .
$$

For the mass correction in the $q$-part of the correlator we use the definition of physical moments given in [12]. The corresponding expression on the contour reads

$$
M_{q}^{p h}(n)=(-1)^{n+1} \frac{1}{2 \pi} \int_{-\pi}^{\pi} C_{q}(\phi) e^{i n \phi} d \phi .
$$

The moments of the massless part $M(n)$ and the mass corrections $M_{q, g}(n)$ are the basic objects which can be calculated theoretically. Our qualitative conclusions about the stability of the running parameters due to the higher order corrections to the $\beta$ and $\gamma$-functions are confirmed by the behavior of the moments. The moments of the massless spectral density given in eq. (17) are stable with regard to inclusion of higher order corrections of the $\beta$-function. This is a direct consequence of the $\beta$-function behavior in consecutive orders of PT along the integration contour plotted in Fig. 1. The moments of the $g$-part given in eq. (18) are less stable with regard to inclusion of higher order corrections of the $\gamma_{g}$-function than the moments of the massless part. The moments of the $q$-part in eq. (19) behave worse in higher orders than the moments of the $g$-part which reflects the slower convergence of the $\gamma_{q}$-function in 
eq. (13) as compared to $\gamma_{g}$-function in eq. (12). In the massless and in the $g$-part the values of the moments are dominated by the second integral in eqs. (17,18) because the values of the integrals with oscillating integrands in eqs. (17,18) decrease for

high order moments. The coefficient functions $C_{q, g}\left(Q^{2}\right)$ depend on the $\gamma_{q, g}$-functions exponentially according to eq. (16) and they are very sensitive to the convergence of the $\gamma_{q, g}$-functions. The exponential dependence of the mass coefficient functions on RG functions is an essentially new feature of the analysis of the $m_{s}^{2}$ corrections as compared to that of the massless part of the $\tau$ lepton decay rate. Such strong dependence requires more accurate treatment of PT series for RG functions.

\section{Determination of $m_{s}$ from $\tau$ decays}

One of the important aims of the analysis of Cabibbo-suppressed $\tau$ decays is the extraction of a numerical value for the mass parameter $m_{s}$. Various observables can be used for this purpose. Here we consider the $\tau$ lepton total decay rate into hadrons. Experimental data for Cabibbo-suppressed hadronic $\tau$ decays are not yet very precise. Contrary to the experimental data the theoretical expressions for the relevant observables related to the moments of the spectral density of the correlator of the hadronic currents are known rather precisely in a sense that the corresponding PT series within OPE are calculated in high (next-to-next-to-leading) orders of expansion in the strong coupling constant. However, this theoretical accuracy is quite formal, because the explicit convergence of PT expressions for the moments is rather slow and the PT series has to be resummed in order to obtain numerical results.

The theoretical expression for the $m_{s}^{2}$ corrections to moments $(k, l)$ of the differential decay rate is given by the contour integral in the complex $q^{2}$-plane

$$
R_{m \tau}^{k l}=\frac{i}{2 \pi} \oint 2\left(1-\frac{q^{2}}{M_{\tau}^{2}}\right)^{2+k}\left(\frac{q^{2}}{M_{\tau}^{2}}\right)^{l} 3\left(\frac{m_{s}^{2} \Pi_{m q}\left(q^{2}\right)}{q^{2}}-\frac{m_{s}^{2}}{M_{\tau}^{2}} \Pi_{m g}\left(q^{2}\right)\right) \frac{d q^{2}}{M_{\tau}^{2}}
$$




$$
=-6\left(\frac{m_{q}^{2}}{M_{\tau}^{2}} A_{k l}+\frac{m_{g}^{2}}{M_{\tau}^{2}} B_{k l}\right)=-6 \frac{m_{s}^{2}}{M_{\tau}^{2}}\left(\omega_{q} A_{k l}+\omega_{g} B_{k l}\right)=-6 \frac{m_{s}^{2}}{M_{\tau}^{2}} F_{k l}
$$

where the superscript $(k, l)$ denotes an integration with additional weight factors which suppress the high $(k>0, l=0)$ and low $(k=0, l>0)$ energy region. The finite order PT expressions for the coefficients $F_{k l}$ can be found in refs. [12, 26]. The analyses performed in ref. [12, 26] in FOPT and CIPT within resummation in the $\overline{\mathrm{MS}}$ scheme have demonstrated that the explicit convergence of the PT series for the moments is slow. A close conclusion about the convergence of PT series in the $\overline{\mathrm{MS}}$ scheme was given in ref. [27]. Therefore, in the present paper we use the contour resummation procedure in the effective scheme and calculate the relevant coefficients $F_{k l}$ in a closed form.

We use the $(0,0)$ moment as our best theoretical estimate for comparison with experiment. Other moments are briefly discussed but are not used in numerical analysis for the strange quark mass determination. The choice of optimal moments is discussed in detail later. In brief, the reasoning is based on analyzing which energy regions saturate the respective moments. Indeed, to choose a special linear combination of moments is equivalent to integrating the running parameters with a special weight function. Whether the corrections to a given linear combination are large or small can depend strongly on the integration region in which the integral of the spectral density is saturated. The change of the running parameters with higher order corrections is big in the region close to the physical cut (at $\phi= \pm \pi$ ) and small in the deep Euclidean region where the functions are fixed by their starting values. If some observables have the regions $\phi= \pm \pi$ strongly suppressed they have no contribution from the perturbative running and, therefore, are very stable with regard to the inclusion of higher order terms of RG functions. The moments of the type $(k, 0)$ represent such observables for large $k$. However, these moments have the contributions from large energy region strongly suppressed and receive relatively large contributions from 
the low energy region that makes them rather nonperturbative. Therefore, the use of the moments $(k, 0)$ with large values of $k$ is not under reliable quantitative control within OPE and perturbation theory, though their use is favorable from the point of view of precision of experimental data. We elaborate on this point later.

\subsection{Relation of the $\overline{\mathrm{MS}}$ mass to effective mass parameters}

Within the effective scheme approach the $\tau$-system is described in its own terms with the mass parameters $m_{q, g}$. In order to obtain a numerical value for $m_{s}$ which can be compared with other determinations we express the natural mass parameters of the $\tau$-system $m_{q, g}$ through the $\overline{\mathrm{MS}}$ mass parameter $m_{s}$. We emphasize that this is only done for purposes of comparison. In principle, observables in the $\tau$-system are best described by their internal parameters $a, m_{q}$ and $m_{g}$. Relations between observables within the $\tau$-system can be found without any reference to the standard $\overline{\mathrm{MS}}$ scheme parameters.

In general, the perturbation theory expression for a given observable in a given renormalization scheme is parametrized by a mass scale and by coefficients of the evolution $(\mathrm{RG})$ functions. In the massless case these are the scale parameter $\Lambda$ and the perturbation theory coefficients of the $\beta$-function (e.g. [36, 37]). In the massive case there are in addition the invariant mass $M$ to be defined in eq. (21) and the coefficients of the $\gamma$-function describing the evolution of the running mass. As in the case of the scale parameter $\Lambda$, the invariant mass $M$ can be defined in different ways. The concrete definition may be fixed by a given asymptotic behavior at large

momenta. This is the way the standard scale parameter $\Lambda_{\overline{\mathrm{MS}}}$ is fixed. We define the invariant mass $M$ in a $\mu$ independent way by writing

$$
M=\frac{m\left(\mu^{2}\right)}{a\left(\mu^{2}\right)^{\gamma_{0} / \beta_{0}}} \exp \left\{-\int_{0}^{a\left(\mu^{2}\right)}\left(\frac{\gamma(\xi)}{\beta(\xi)}-\frac{\gamma_{0}}{\beta_{0} \xi}\right) d \xi\right\} .
$$

Note that $M$ is renormalization group invariant. That means that if $m\left(\mu^{2}\right)$ is redefined 
by some RG transformation the change is absorbed by the corresponding change of the $\gamma$-function so that $M$ remains invariant up to the order in the coupling which has been taken into account. The RG invariance of $M$ can be used to relate running masses defined in different renormalization schemes. After squaring eq. (21) we find the relation between two mass definitions in different schemes with the $\gamma$-functions $\gamma(a)$ and $\gamma^{\prime}(a)$ expressed in terms of the same coupling $a$. In our analysis we use the effective coupling $a$ as it is defined in eq. (幽 and relate $m_{n}^{2}(n=q, g)$ to $m^{\prime 2}$ by

$$
m_{n}^{2}\left(\mu^{2}\right)=m_{s}^{\prime 2}\left(\mu^{2}\right) \exp \left\{-2 \int_{0}^{a\left(\mu^{2}\right)} \frac{\gamma^{\prime}(\xi)-\gamma_{n}(\xi)}{\beta(\xi)} d \xi\right\} .
$$

Here $m_{s}^{\prime 2}$ is the standard $\overline{\mathrm{MS}}$ scheme mass but with the evolution function $\gamma^{\prime}(a)$ expressed through the effective coupling. Eq. (22) relates two mass parameters defined in different renormalization schemes but expressed through the same coupling. Therefore the standard $\gamma$-function of the $\overline{\mathrm{MS}}$ mass should be rewritten in terms of the effective parameter $a$ before the use in eq. (22). It is also possible to use eq. (21) written in the $\overline{\mathrm{MS}}$ scheme and in the effective scheme. Then one determines the relation between $m_{q, g}$ and $m_{s}$ by eliminating the invariant mass $M$ directly

$$
\begin{aligned}
\frac{m_{n}^{2}\left(\mu^{2}\right)}{m_{s}^{2}\left(\mu^{2}\right)}= & \left(\frac{a_{n}\left(\mu^{2}\right)}{a_{\overline{\mathrm{MS}}}\left(\mu^{2}\right)}\right)^{\frac{2 \gamma_{0}}{\beta_{0}}} \times \exp \left\{-2 \int_{0}^{a \overline{\mathrm{MS}}\left(\mu^{2}\right)}\left(\frac{\gamma_{\overline{\mathrm{MS}}}(\xi)}{\beta_{\overline{\mathrm{MS}}}(\xi)}-\frac{\gamma_{0}}{\beta_{0} \xi}\right) d \xi\right\} \\
& \times \exp \left\{2 \int_{0}^{a_{n}\left(\mu^{2}\right)}\left(\frac{\gamma_{n}(\xi)}{\beta_{\mathrm{eff}}(\xi)}-\frac{\gamma_{0}}{\beta_{0} \xi}\right) d \xi\right\} .
\end{aligned}
$$

In our case the effective couplings $a_{n}, n=q, g$ are equal to the same coupling $a$ for both masses $m_{q, g}$. The two procedures described above lead to close numerical values for the coefficients $\omega_{q, g}$ relating the effective mass parameters $m_{q, g}$ to the $\overline{\mathrm{MS}}$ scheme mass $m_{s}$. The difference of numerical values for $\omega_{q}$ and $\omega_{g}$ obtained from the two procedures described by eqs. (22) and (23) turns out to be less than $3 \%$ which is the residual scheme dependence of the results. Eqs. (22,23) allow us to express the internal parameters $m_{q, g}$ through the standard $\overline{\mathrm{MS}}$ scheme parameter $m_{s}$ (see 
eqs. (6, (1) and $(9,10))$. Finally we find for the coefficients $\omega_{q, g}$ relating the effective mass parameters $m_{q, g}$ to the reference $\overline{\mathrm{MS}}$ mass $m_{s}$

$$
\begin{aligned}
& m_{q}=\omega_{q} m_{s}, \quad m_{g}=\omega_{g} m_{s} \\
& \omega_{q}=1.73 \pm 0.04, \quad \omega_{g}=1.42 \pm 0.03
\end{aligned}
$$

The numerical values for the coefficients $\omega_{q, g}$ are not close to unity which shows that perturbation theory corrections for observables in the $\tau$-system are rather large in the $\overline{\mathrm{MS}}$ scheme. The FOPT expansion for the coefficients $\omega_{q, g}$ has been obtained in ref. [12]. This expansion converges slowly which forces us to use the exact RG conversion given in eqs. (22,23).

\subsection{Power corrections from dimension $D=4$ condensate terms}

For the determination of $m_{s}$ one needs not all $D=4$ condensate corrections to the theoretical expression for the $\tau$ lepton decay rate but only those that enter the difference

$$
\delta R_{\tau}^{k l}=\frac{R_{\tau s=0}^{k l}}{\left|V_{u d}\right|^{2}}-\frac{R_{\tau s=1}^{k l}}{\left|V_{u s}\right|^{2}}
$$

Here $R_{\tau s=0,1}^{k l}$ is defined as

$$
R_{\tau s=0,1}^{k l}=\int_{0}^{M_{\tau}^{2}} d s\left(1-\frac{s}{M_{\tau}^{2}}\right)^{k}\left(\frac{s}{M_{\tau}^{2}}\right)^{l} \frac{d R_{\tau s=0,1}}{d s}
$$

and $d R_{\tau s=0,1} / d s$ is the differential $\tau$-decay rate into the final hadronic states with the strangeness 0,1 and the energy $\sqrt{s}$. In the theoretical expression for the difference $\delta R_{\tau}^{k l}$ we neglect terms of the order $m_{s}^{3} / M_{\tau}^{3}$, set the $u$ - and $d$-quark masses to zero, and retain only the most important term linear in $m_{s}$ (cf. refs. [26, 27]). Within OPE the coefficient of this term is given by the quark condensate. The final result reads

$$
\delta R_{\tau}^{k l}=N_{c} S_{E W}\left(6 \frac{m_{s}^{2}}{M_{\tau}^{2}} F_{k l}-4 \pi^{2} \frac{m_{s}}{M_{\tau}} \frac{\langle\bar{s} s\rangle}{M_{\tau}^{3}} T_{k l}\right)
$$


where $N_{c}=3, m_{s} \equiv m_{s}\left(M_{\tau}^{2}\right)$, and $S_{E W}=1.0194$ describes electroweak corrections [38, 39. The coefficient function of the $D=4$ local operator $m_{s} \bar{s} s$ is taken in the leading order of perturbation theory expansion within OPE. In this approximation for the coefficient function the quantities $T_{k l}$ multiplying the quark condensate are given by the expression

$$
T_{k l}=2\left(\delta_{l, 0}(k+2)-\delta_{l, 1}\right)
$$

The numerical values for the first few coefficients $T_{k l}$ read

$$
T_{00}=4, \quad T_{10}=6, \quad T_{20}=8, \quad T_{01}=-2, \quad T_{11}=-2 .
$$

These results agree with the leading order expressions for the coefficients given in ref. [27]. We have set up all ingredients necessary for the evaluation of eq. (27). Table 1 gives the coefficients of the $q$ - and $g$-mass parameters in eq. (20).

\section{Numerical analysis and the choice of moments}

Having theoretical expressions for all moments we have still to optimize the choice for comparison with experiment and extraction of the numerical value for the strange quark mass. As was mentioned above the theoretical expressions for the moments $(0, l), l>0$ are more reliable from the point of view of perturbation theory than those for the moments $(k, l)$ with nonzero $k$ (the detailed discussion of this point is given below). However, experimental precision is worse for the moments $(0, l)$ with large $l$ because such moments are saturated by the contributions of many-particles hadronic states which is difficult to measure (see Table 2) . Note that some manyparticles hadronic state contributions in the experimental data (for instance, $K 4 \pi$ contribution) are represented by a result of Monte-Carlo simulation rather than direct measurements. Therefore, we use the moment $(0,0)$ as our best choice from both 


\begin{tabular}{||c||c|c|c||c|c|c||}
\hline \hline$(k, l)$ & $A_{k l}^{\mathrm{LO}}$ & $A_{k l}^{\mathrm{NLO}}$ & $A_{k l}^{\mathrm{NNLO}}$ & $B_{k l}^{\mathrm{LO}}$ & $B_{k l}^{\mathrm{NLO}}$ & $B_{k l}^{\mathrm{NNLO}}$ \\
\hline$(0,0)$ & 1.361 & 1.445 & 1.434 & 0.523 & 0.601 & 0.625 \\
$(1,0)$ & 1.568 & 1.843 & 1.976 & 0.441 & 0.552 & 0.601 \\
$(2,0)$ & 1.762 & 2.282 & 2.646 & 0.390 & 0.530 & 0.607 \\
\hline$(0,1)$ & -0.207 & -0.398 & -0.542 & 0.082 & 0.050 & 0.025 \\
\hline \hline
\end{tabular}

Table 1: Coefficients of eq. (20)

experimental and theoretical point of view. The theoretical expression for this moment exhibits a rather good convergence in consecutive orders of perturbation theory and the accuracy of experimental data for it is still acceptable in comparison with higher $(0, l)$ moments. Note that the perturbative convergence is the main concern of the theoretical analysis in both massless and massive cases. In the massless case the nonperturbative corrections are small if factorization is used for the four-quark condensates [11, 40, 41].

\subsection{Numerical value for the strange quark mass}

The coefficients $A_{k l}$ are related to the $q$-part of the correlator. This part contains contributions from spin 0 and spin 1 particles. The spin 0 piece is prone to possible nonperturbative contributions of (direct) instantons and perturbation theory expansions are expected to break down in low orders in this channel. However, this is only a general expectation without strict quantitative estimates of applicability of PT. The coefficients $B_{k l}$ are related to the $g$-part of the correlator. The $g$-part contains only contributions of spin 1 particles. Nonperturbative contributions of (direct) instantons are forbidden in this channel by symmetry considerations. The coefficient $F_{k l}$ of $m_{s}^{2}$ 
term in eq. (27) is combined according to eq. (20)

$$
F_{k l}=\omega_{q} A_{k l}+\omega_{g} B_{k l}
$$

As for the linear in $m_{s}$ term in eq. (27) we calculate its numerical coefficient using a phenomenological value for the quark condensate. We use the relation [42, 43, 44,

$$
\langle\bar{s} s\rangle=(0.8 \pm 0.2)\langle\bar{u} u\rangle
$$

and the numerical value $\langle\bar{u} u\rangle=-(0.23 \mathrm{GeV})^{3}$ which coincides with the standard value (see e.g. [27]). Substituting all necessary quantities into eq. (27) we arrive at the defining equation for $X=m_{s} /(130 \mathrm{MeV})$

$$
\frac{1}{N_{c} S_{E W}}\left(\frac{M_{\tau}}{130 \mathrm{MeV}}\right)^{2} \delta R_{\tau}^{k l}=X\left(6 F_{k l} \cdot X+0.936 \cdot T_{k l}\right) .
$$

The dimension-four term contributes appreciably to the total theoretical result for different moments. For the moments $(k, 0)$ its relative contribution increases with $k$ for the first few moments. We use only the moment $(0,0)$ for which the dimensionfour contribution gives about $16 \%$ of the total result. The coefficient function of the dimension-four contribution converges well in the perturbative expansion within OPE. We do not take the PT corrections to the coefficient function of the dimensionfour contribution into account (see e.g. [27]) because of the large uncertainty in the numerical value of the strange quark condensate in eq. (31).

For extraction of the numerical value for the strange quark mass we use the experimental data obtained by the ALEPH collaboration [2]. The results for $m_{s}\left(M_{\tau}^{2}\right)$ from different moments $\delta R_{\tau}^{k l}$ are given in Table 2. For the determination of $m_{s}$ we use only the moment $(0,0)$ as the most reliable one from the perturbation theory point of view. A detailed discussion of the justification for our choice is given later.

The final relation for determining the central value of the strange quark mass from the data on Cabibbo-suppressed $\tau$ decays reads

$$
\frac{1}{N_{c} S_{E W}}\left(\frac{M_{\tau}}{130 \mathrm{MeV}}\right)^{2}\left(\delta R_{\tau}^{00}\right)^{\exp }=24.1=X(20.2 X+3.74) .
$$




\begin{tabular}{|c|c|c|}
\hline$(k, l)$ & $\left(\delta R_{\tau}^{k l}\right)^{\exp }$ & $m_{s}\left(M_{\tau}^{2}\right) \mathrm{MeV}$ \\
\hline$(0,0)$ & $0.394 \pm 0.137$ & 130 \\
$(1,0)$ & $0.383 \pm 0.078$ & 111 \\
$(2,0)$ & $0.373 \pm 0.054$ & 95 \\
\hline
\end{tabular}

Table 2: Results for $m_{s}\left(M_{\tau}\right)$ obtained from different moments of $\delta R_{\tau}$

The result is $X=m_{s} /(130 \mathrm{MeV})=1.00 \ldots$ with the accuracy of two decimal places. This leads to our final prediction for the strange quark mass at $M_{\tau}$

$$
m_{s}\left(M_{\tau}^{2}\right)=130 \pm 27_{\exp } \pm 3_{\langle\bar{s} s\rangle} \pm 6_{\text {th }} \mathrm{MeV} .
$$

Note that this result is obtained with the numerical value for the effective coupling $a\left(M_{\tau}^{2}\right)=0.1445$ extracted from the $\tau$ decay rate into nonstrange particles within the effective scheme resummation procedure described in ref. [19]. The reference numerical value of the $\overline{\mathrm{MS}}$ scheme coupling constant is $\alpha_{s}\left(M_{\tau}^{2}\right)=0.343$.

We also give the value for $m_{s}(1 \mathrm{GeV})$ obtained from $m_{s}\left(M_{\tau}^{2}\right)$ in eq. (34) after four-loop running in the $\overline{\mathrm{MS}}$ scheme

$$
m_{s}(1 \mathrm{GeV})=176 \pm 37_{\exp } \pm 4_{\langle\bar{s} s\rangle} \pm 9_{\text {th }} \mathrm{MeV}
$$

The numerical value of the strange quark mass at the scale $1 \mathrm{GeV}$ depends on the way the evolution is performed because of the truncation of the PT series for the RG functions. This difference reflects the residual scheme dependence of the evolution.

The numerical value for the invariant mass $M$ defined in eq. (21) and calculated in the $\overline{\mathrm{MS}}$ scheme with $\alpha_{s}\left(M_{\tau}^{2}\right)=0.343$ reads

$$
M=312 \pm 65_{\exp } \pm 7_{\langle\bar{s} s\rangle} \pm 14_{\text {th }} \mathrm{MeV} .
$$

This value can be used for comparison of the results of the strange quark mass determination obtained from different theoretical calculations and experimental data. If 
the effective scheme is used for determination of the numerical value for the invariant mass $M$ from eq. (21) the result is slightly different that reflects the residual scheme dependence due to truncation of PT series for $\beta$ - and $\gamma$-functions.

Results presented in eqs. (35, 34) are the main new numerical results of the paper. Another new results is a formulation of the problem in an effective scheme and the development of all the necessary techniques for phenomenological applications.

Note that the uncertainty of the final result is smaller than the difference between the results of mass extraction from the zero and second moment (first and the last lines of Table 21). As we have mentioned before and explain in detail later on we do not consider the results obtained from the high moments as reliable which means that the theoretical uncertainties of the numerical mass value $m_{s}^{(1)}=111 \mathrm{MeV}$ (from the first moment) and $m_{s}^{(2)}=95 \mathrm{MeV}$ (from the second moment) are expected to be much larger than the uncertainty of our optimal choice - the zeroth moment. Therefore, the results of all determinations are consistent but the uncertainty of higher moments is large. Our view is that after accounting for high dimension condensates the second and third determination will change (while the first one does not) and may lie within the error bars. Since there is no reason to expect the effect of high-dimension condensates to be negligible the requirement that the first and third determination are the same implicitly implies that high-dimension condensates are negligible which is just an additional assumption (without any justification). Because we do not use these moments in our determination we do not quantitatively discuss these uncertainties.

\subsection{Comparison with other results}

Our final result for the numerical coefficient in front of the $m_{s}^{2}$ correction in eq. (33) agrees with the estimate obtained from the analysis in the $\overline{\mathrm{MS}}$ scheme $[26$. In the present paper we find $20.2 \pm 1.8$ for the coefficient of the $m_{s}^{2}$ correction while the 
final result of ref. [26] with the conservative estimate of the error bar is $18.1 \pm 4.8$. The final uncertainty of the result obtained in ref. [26] was determined from both resummed and FOPT analyses. If only the CIPT analysis in the $\overline{\mathrm{MS}}$ scheme is used then one obtains the value $18.1 \pm 2.6$ which has a smaller uncertainty [26]. The present value $20.2 \pm 1.8$ results from the analysis in the effective scheme with stricter criteria of convergence and more conservative error estimate; for instance, in the final result for $F_{k l}$ we have doubled the error for coefficients $\omega_{q, g}$ from eqs. (24). However, the interpretation of the perturbation series in a closed manner (resummation in the effective scheme) allows one to reduce the uncertainty of theoretical expressions in comparison with the previous analysis in the $\overline{\mathrm{MS}}$ scheme performed in ref. [26]. This leads to an essential reduction of the theoretical part of the error in the extracted numerical value for the strange quark mass.

The analysis of mass corrections in the $\overline{\mathrm{MS}}$ scheme was also done in ref. [27] where a very accurate account for known PT corrections to the coefficient functions of nonPT corrections was given. The direct comparison of our results with ref. 27] is not simple because different representations have been chosen for the observables. Also some approximations have been used which prevents us from directly comparing with the results of the present paper at intermediate stages. The final results for the mass extracted from the zeroth order moment is rather close to the result of ref. [27]. Still in general the results of refs. [26, 27] are rather close to each other concerning use of scheme while the change of the scheme was the main reason for our analysis as for comparison with ref. [26]. The analysis of the present paper also extends the analysis of scale dependence which has been done in ref. [27].

The resulting values of the strange quark mass are close to the earlier estimates obtained in refs. [45, 46, 47, 48] with the use of less accurate theoretical input formulas and less precise experimental data. The recent analysis based on (pseudo)scalar 
correlators with a thorough parameterization of experimental data gives a value close to ours [49, 50]. In a lattice calculation of the numerical value for the strange quark mass [51, 52] the theoretical input is of nonperturbative nature. The recent results obtained in lattice calculations are smaller than our value for $m_{s}$ but still inside the error bars as given in eq. (34).

\subsection{Choice of moments}

High moments with the weight function $\left(1-s / M_{\tau}^{2}\right)^{k}$ (large values of $k$ ) are saturated by nonperturbative (infrared, or low-energy) contributions because the perturbative region of integration (large energy) is suppressed. On the experimental side this means that such moments are saturated by the contributions of low lying resonances. In the considered case this is the contribution of the $K$ meson in spin 0 channel and of the system $K_{1}(1270)-K_{1}(1400)$ in the spin 1 channel. The low-energy contributions can be accurately measured that makes the moments obtained from the experimental data rather precise. On the theoretical side, within the OPE calculation of the correlator the infrared sensitivity of high- $k$ moments means that the PT contribution to such moments is suppressed and the moments are saturated by the contributions of vacuum condensates of high-dimension operators. Implicitly this is seen in a poor convergence of the PT series for such moments which is demonstrated in 12. To obtain an accurate numerical value for such moments one has to include vacuum condensates of high-dimension operators. However, numerical values for vacuum condensates of the local operators with dimension larger than six are completely unknown and are usually neglected in the $\tau$-system analyses. Therefore, high moments with the weight function $\left(1-s / M_{\tau}^{2}\right)^{k}$ have an uncontrollable admixture of high-dimension condensates that makes them strongly nonperturbative and, therefore, unreliable for practical PT applications. Indeed, the moments $(k, 0)$ obtain contributions from all condensates 
up to dimension $D=2 k+8$ the numerical values of which are unknown for large $k$. Because on the experimental side this contribution corresponds to the contribution of low-lying resonance which is not described by PT it seems unjustified to neglect the contributions from high-dimension condensates and use only the PT expressions (supplemented by condensates only up to dimension 6 which are available) for large $k$ moments. In the analysis presented in ref. [27 the contributions from high-dimension condensates (with $D>6$ ) were regarded as an additional theoretical uncertainty. Note, however, that the high-dimension condensates contribute quite differently to moments $(k, 0)$ and $(0, l)$ because the integrals corresponding to these moments are saturated by contributions of different energy regions. The $(0, l)$ moments for large $l$ are saturated at large energies (of order $M_{\tau}$ ) and nonPT contributions described by the high-dimension condensates within OPE are small. In the leading order only one condensate is picked up by integration for a $(0, l)$ moment (assuming that the factor $\left(1-s / M_{\tau}^{2}\right)^{2}$ is removed from the rate). On the contrary, the $(k, 0)$ moments for large $k$ are saturated at small energies which correspond to the region of strong coupling: these moments are dominated by the resonances. Therefore the $(k, 0)$ moments are definitely nonperturbative for large $k$. Within OPE paradigm this means that the total contribution of high-dimension condensates is large compared to perturbative contribution. Indeed, all condensates with dimension up to $D=2 k+8$ contribute to $(k, 0)$ moments and arrange themselves in a way to reproduce contributions of the low-lying resonances according to the standard phenomenology within OPE. This situation is clearly seen in exactly solvable models where all power corrections are known. In QCD, however, numerical values of high-dimension condensates are not known and the total contribution of high-dimension condensates cannot be quantitatively analyzed for large $k$ moments though qualitative arguments are rather transparent. These reasons forced us to use only the $(0,0)$ moment as the most re- 
liable from the theoretical point of view even despite the fact that the experimental precision for high $k$ moments is better.

\section{Conclusion}

We have considered the $m_{s}^{2}$-corrections in a QCD-based description of the $\tau$-system. We use a natural effective scheme well suited for a contour improved perturbation theory analysis. The quality of our results is determined by the PT expansions of the effective $\beta$ - and $\gamma$-functions which are the only perturbative objects in our analysis. The $\gamma_{q}$-function already shows an asymptotic growth at NNLO while the $\beta$ - and $\gamma_{g}$-functions still 'converge' up to this order. In our discussion of the $\mathrm{N}^{3} \mathrm{LO}$ terms of PT series for these functions (which depend on the unknown parameter $k_{3}$ ) we found strong indications for asymptotic growth at this order. This shows that the ultimate theoretical limit of FOPT precision is already reached for the set of observables in the $\tau$-system. This is not yet an actual problem of QCD because of the insufficient precision of the experimental data on Cabibbo-suppressed $\tau$ decays especially regarding the differential $\tau$ decay rate. The experimental situation may, however, improve soon. Then our procedure of using the effective scheme description of the $\tau$-system opens the possibility of high precision tests of QCD independently of an explicit convergence of PT series in the $\overline{\mathrm{MS}}$ scheme. In this field an effective scheme approach can show its real power because then the main source of theoretical uncertainty, the relation of the internal mass parameters $m_{q}, m_{g}$ to the $\overline{\mathrm{MS}}$ parameter $m_{s}$, will be eliminated. Note that for a QCD test within our approach it is necessary to relate four $\tau$-observables to each other because the three parameters $a, m_{g}$ and $m_{q}$ have to be fixed.

Our result for the strange quark mass is $m_{s}\left(M_{\tau}^{2}\right)=130 \pm 27_{\exp } \pm 9_{\text {th }} \mathrm{MeV}$ where

we have combined the pure theoretical error which is basically determined by the 
truncation of PT series for effective RG functions and the error due to the strange quark condensate into one number linearly. After running to the scale $1 \mathrm{GeV}$ we obtain $m_{s}(1 \mathrm{GeV})=176 \pm 37_{\exp } \pm 13_{\text {th }} \mathrm{MeV}$. This is consistent with the previous

results where the resummation was done in the $\overline{\mathrm{MS}}$ scheme [26]. The large part of the theoretical uncertainty of our result for the PT coefficient of $m_{s}^{2}$ term comes from re-expressing the effective quantities in terms of the $\overline{\mathrm{MS}}$ scheme parameters. The advantage of the procedure presented here is that the estimate of the accuracy is not based on the decomposition of the result into terms coming from corrections to the correlator in the $\overline{\mathrm{MS}}$ scheme. This decomposition seems unnatural in resummed perturbation theory as it introduces an additional uncertainty related to the convergence of the series for the correlator in the $\overline{\mathrm{MS}}$ scheme every term of which is given by a closed expression resulting from the resummation along the contour. Within the effective scheme approach all sources of uncertainty are collected into the effective $\beta$ - and $\gamma$-functions which are the only PT quantities entering into the analysis that provides a solid ground for estimating the accuracy of theoretical expressions.

\section{Acknowledgments}

A.A. Pivovarov thanks K.G. Chetyrkin for discussion and correspondence. The work is partially supported by the Russian Fund for Basic Research under contracts 99-01-00091 and 01-02-16171 and by the Volkswagen Foundation under contract No. I/73611.

\section{Appendix}

We give the expressions for the effective $\beta$ - and $\gamma$-function coefficients in terms of the standard $\overline{\mathrm{MS}}$ scheme results. In this Appendix the coefficients of $\beta$ - and $\gamma$-functions 
without upper index stand for $\overline{\mathrm{MS}}$ quantities while the coefficients with upper index "eff" denote the coefficients of the effective functions.

The coefficients of the effective $\beta$-function (8) are given by

$$
\begin{aligned}
& \beta_{0}^{\mathrm{eff}}=\beta_{0}, \quad \beta_{1}^{\mathrm{eff}}=\beta_{1} \\
& \beta_{2}^{\mathrm{eff}}=\beta_{2}-k_{1} \beta_{1}+\left(k_{2}-k_{1}^{2}\right) \beta_{0} \\
& \beta_{3}^{\mathrm{eff}}=\beta_{3}-2 k_{1} \beta_{2}+k_{1}^{2} \beta_{1}+\left(2 k_{3}-6 k_{2} k_{1}+4 k_{1}^{3}\right) \beta_{0} .
\end{aligned}
$$

For the effective $\gamma$-function coefficients of eqs. (9, 10) in terms of the $\overline{\mathrm{MS}}$ scheme $\beta$ and $\gamma$-function coefficients we find

$$
\begin{aligned}
\gamma_{n 0}^{\text {eff }}= & \gamma_{0}, \quad \gamma_{n 1}^{\text {eff }}=\gamma_{1}-k_{1} \gamma_{0}+\frac{1}{2} k_{n 0} \beta_{0}, \\
\gamma_{n 2}^{\text {eff }}= & \gamma_{2}-2 k_{1} \gamma_{1}+\left(-k_{2}+2 k_{1}^{2}\right) \gamma_{0}+\frac{1}{2} k_{n 0} \beta_{1}+\left(-k_{1} k_{n 0}+k_{n 1}-\frac{1}{2} k_{n 0}^{2}\right) \beta_{0}, \\
\gamma_{n 3}^{\text {eff }}= & \gamma_{3}-3 k_{1} \gamma_{2}+\left(-2 k_{2}+5 k_{1}^{2}\right) \gamma_{1}+\left(-k_{3}+5 k_{2} k_{1}-5 k_{1}^{3}\right) \gamma_{0} \\
& +\frac{1}{2} k_{n 0} \beta_{2}+\left(-\frac{3}{2} k_{1} k_{n 0}+k_{n 1}-\frac{1}{2} k_{n 0}^{2}\right) \beta_{1} \\
& +\left(-k_{2} k_{n 0}+\frac{5}{2} k_{1}^{2} k_{n 0}-3 k_{1} k_{n 1}+\frac{3}{2} k_{1} k_{n 0}^{2}+\frac{3}{2} k_{n 2}-\frac{3}{2} k_{n 1} k_{n 0}+\frac{1}{2} k_{n 0}^{3}\right) \beta_{0} .
\end{aligned}
$$

Here $k_{n j}$ with $n=q, g$ stand for the coefficients of eq. (6) if $n=g\left(k_{g 0}=5 / 3\right)$ and for the coefficients of eq. (7) if $n=q\left(k_{q 0}=7 / 3\right)$. Numerical values for all necessary

coefficients in the $\overline{\mathrm{MS}}$ scheme have been collected in [12] where further references to original papers can be found.

\section{References}

[1] Particle Data Group, Review of Particle Properties, Eur. Phys. J. C3 (1998) 1.

[2] ALEPH collaboration, Z. Phys. C76 (1997) 15;

Eur. Phys. J. C4 (1998) 409; ibid C11 (1999) 599.

[3] OPAL collaboration, Eur. Phys. J. C7 (1999) 571. 
[4] C. Bernard, A. Duncan, J. LoSecco and S. Weinberg, Phys. Rev. D12 (1975) 792;

E. Poggio, H. Quinn and S. Weinberg, Phys. Rev. D13 (1976) 1958.

[5] R. Shankar, Phys. Rev. D15 (1977) 755.

[6] K.G. Chetyrkin, N.V. Krasnikov and A.N. Tavkhelidze, Phys. Lett. 76B (1978) 83.

[7] K. Schilcher and M.D. Tran, Phys. Rev. D29 (1984) 570.

[8] E. Braaten, Phys. Rev. Lett. 53 (1988) 1606; Phys. Rev. D39 (1989) 1458.

[9] S. Narison and A. Pich, Phys. Lett. B211 (1988) 183.

[10] A.A. Pivovarov, Sov. J. Nucl. Phys. 54 (1991) 676.

[11] E. Braaten, S. Narison and A. Pich, Nucl. Phys. B373 (1992) 581.

[12] J.G. Körner, F. Krajewski and A.A. Pivovarov, Eur. Phys. J. C12 (2000) 461; ibid C14 (2000) 123.

[13] K.G. Chetyrkin, R. Harlander and J.H. Kühn, TTP-99-42; hep-ph/9910345.

[14] V.I. Zakharov, Prog. Theor. Phys. Suppl. 131 (1998) 107.

[15] N.V. Krasnikov and A.A. Pivovarov, Mod. Phys. Lett. A11 (1996) 835; hepph/9510207.

[16] A.A. Pivovarov, Nucl. Phys. Proc. Suppl. 64 (1998) 339; hep-ph/9708461; Phys. Atom. Nucl. 62 (1999) 1924; Sov. J. Nucl. Phys. 52 (1990) 372.

[17] A.A. Pivovarov, Z. Phys. C53 (1992) 461; Nuovo Cim. 105A (1992) 813.

[18] F. Le Diberder and A. Pich, Phys. Lett. B286 (1992) 147. 
[19] S. Groote, J.G. Körner and A.A. Pivovarov, Phys. Lett. B407 (1997) 66; Mod. Phys. Lett. A13 (1998) 637.

[20] G. Grunberg, Phys. Lett. B95 (1980) 70, Erratum-ibid B110 (1982) 501.

[21] N.V. Krasnikov, Nucl. Phys. B192 (1981) 497.

[22] A.L. Kataev, N.V. Krasnikov and A.A. Pivovarov, Phys. Lett. B107 (1981) 115; Nucl. Phys. B198 (1982) 508, Erratum-ibid B490 (1997) 505.

[23] A. Dhar and V. Gupta, Phys. Rev. D29 (1984) 2822.

[24] S.J. Brodsky, J.R. Pelaez and N. Toumbas, Phys. Rev. D60 (1999) 037501.

[25] S. Groote, J.G. Körner, A.A. Pivovarov and K. Schilcher, Phys. Rev. Lett. 79 (1997) 2763.

[26] K.G. Chetyrkin, J.H. Kühn and A.A. Pivovarov, Nucl. Phys. B533 (1998) 473.

[27] A. Pich and J. Prades, JHEP 9806 (1998) 013; ibid 9910 (1999) 004.

[28] K.G. Chetyrkin, J.H. Kühn and A. Kwiatkowski, Phys. Rep. 277 (1996) 189.

[29] S.G. Gorishny, A.L. Kataev and S.A. Larin, Phys. Lett. B259 (1991) 144.

[30] L.R. Surguladze and M.A. Samuel, Phys. Rev. Lett. 66 (1991) 560, 2416(E), Phys.Rev. D44 (1991) 1602.

[31] K.G. Chetyrkin, Phys. Lett. B391 (1997) 402.

[32] T. van Ritbergen, J.A.M. Vermaseren and S.A. Larin, Phys. Lett. B400 (1997) 379.

[33] K.G. Chetyrkin, Phys. Lett. B404 (1997) 161. 
[34] T. van Ritbergen, J.A.M. Vermaseren and S.A. Larin, Phys. Lett. B405 (1997) 327.

[35] J.G. Körner, F. Krajewski and A.A. Pivovarov, MZ-TH-00-03, hep-ph/0002166.

[36] P.M. Stevenson, Phys. Rev. D23 (1981) 2916.

[37] J. Kubo, S. Sakakibara and P.M. Stevenson, Phys. Rev. D29 (1984) 1682.

[38] W.J. Marciano and A. Sirlin, Phys. Rev. Lett. 61 (1988) 1815.

[39] E. Braaten and C.S. Li, Phys. Rev. D42 (1990) 3888.

[40] M.A. Shifman, A.I. Vainshtein and V.I. Zakharov, Nucl. Phys. B147 (1979) 385.

[41] K.G. Chetyrkin and A.A. Pivovarov, Nuovo Cim. 100A (1988) 899.

[42] C. Becchi, S. Narison, E. de Rafael and F.J. Yndurain, Z. Phys. C8 (1981) 335.

[43] A.A. Ovchinnikov and A.A. Pivovarov, Phys. Lett. B163 (1985) 231;

N.V. Krasnikov and A.A. Pivovarov, Nuovo Cim. 81A (1984) 680.

[44] Y. Chung et al., Z. Phys. C25 (1984) 151; H.G. Dosch, M. Jamin and S. Narison, Phys. Lett. B220 (1989) 251.

[45] S. Narison and E. de Rafael, Phys. Lett. B103 (1981) 57.

[46] J. Gasser and H. Leutwyler, Phys. Rept. 87 (1982) 77.

[47] A.L. Kataev, N.V. Krasnikov and A.A. Pivovarov, Phys. Lett. B123 (1983) 93; Nuovo Cim. 76A (1983) 723.

[48] S.G. Gorishnii, A.L. Kataev and S.A. Larin, Phys. Lett. B135 (1984) 457.

[49] M. Jamin and M. Munz, Z. Phys. C66 (1995) 633. 
[50] K.G. Chetyrkin, D. Pirjol and K. Schilcher, Phys. Lett. B404 (1997) 337.

[51] ALPHA and UKQCD Collaboration (J. Garden et al.), Nucl. Phys. B571 (2000) 237.

[52] ALPHA Collaboration (M. Guagnelli et al.), Nucl. Phys. B560 (1999) 465. 